\title{
Hospital Information System in the Context of the Use of Electronic Medical Record in Burundi
}

\author{
Munezero Florence ${ }^{1, *}$, Ahanhanzo Glele Yolaine ${ }^{2}$, Sossa Jerome Charles ${ }^{2}$, Bazira Leodegard ${ }^{3}$ \\ ${ }^{1}$ Direction of Research, National Institute of Public Health, Bujumbura, Burundi \\ ${ }^{2}$ Regional Institute of Public Health, Ouidah, Benin \\ ${ }^{3}$ Faculty of Medicine, University of Burundi, Bujumbura, Burundi
}

Received January 23, 2021; Revised March 22, 2021; Accepted April 18, 2021

\section{Cite This Paper in the following Citation Styles}

(a): [1] Munezero Florence, Ahanhanzo Glele Yolaine, Sossa Jerome Charles, Bazira Leodegard, "Hospital Information System in the Context of the Use of Electronic Medical Record in Burundi," Universal Journal of Public Health, Vol. 9, No. 2, pp. 94 - 101, 2021. DOI: 10.13189/ujph.2021.090208.

(b): Munezero Florence, Ahanhanzo Glele Yolaine, Sossa Jerome Charles, Bazira Leodegard (2021). Hospital Information System in the Context of the Use of Electronic Medical Record in Burundi. Universal Journal of Public Health, 9(2), 94 - 101. DOI: 10.13189/ujph.2021.090208.

Copyright $\bigcirc 2021$ by authors, all rights reserved. Authors agree that this article remains permanently open access under the terms of the Creative Commons Attribution License 4.0 International License

\begin{abstract}
In order to strengthen the Information System, the medical record project has been initiated since 2014. The objective of this study was to assess the Hospital Information System in the context of using the Electronic Medical Record (EMR) to highlight the factors to be strengthened. This is a cross-sectional survey carried out in October 2019 in 11 hospitals equipped with a Hospital Information System based on OpenClinic GA software. The evaluation was carried out using the Health Metrology Network (HMN) tool covering the components of the Health Information System. Data entry and analysis were carried out with the Excel tool transposed to the HMN tool format. The calculation of the scores and the thresholds used are based on the same HMN tool. The score was $61 \%$ for resources, $60 \%$ for indicators, $41 \%$ for data sources, $51 \%$ for data management, $55 \%$ for information product and $67 \%$ for information dissemination and use. According to the period of time when the EMR was first used, the 7 hospitals computerized in 2017 had an overall score of $57 \%$ compared to $53 \%$ of the 4 hospitals computerized in 2015 $(p=0.7055)$. District hospitals had an overall score of $61 \%$ while regional hospitals had an overall score of $50 \%$ and national hospitals had an overall score of $52 \%(\mathrm{p}=0.1441)$. In conclusion, the results show that two components namely source of the data and data management have shortcomings. Corrective strategies should be considered to support the ongoing hospital information system strengthening reforms.
\end{abstract}

Keywords Information System, Hospital, Electronic Medical Record

\section{Introduction}

The role of the hospital is to carry out health care and administrative activities, cooperating together for the specific purpose of ensuring the quality of health care and services provided to the population. Thus, the management of hospital services requires relevant, reliable and timely information to enable appropriate and timely decision-making [1][2]. Therefore, the hospital's organization requires a Hospital Information System (HIS) which must be able to gather and process medical and administrative data and provide information with high added value to all the hospital's internal and external stakeholders [3][4].

The patient record, an essential component of the HIS, contributes to the coordination of multidisciplinary care and to the quality of care. Optimal patient care requires, among other things, the use of the electronic medical record to ensure close coordination of services provided by several providers and efficient management of all data generated, exchanged and stored [5]. It is a communication and information tool between providers on the one hand and between providers and patients on the 
other. It allows the patient's hospital journey to be monitored and understood, and is a key element in the quality of care by allowing continuity of care within the framework of multi-professional and multi-disciplinary management [2].

With the emergence of information and communication technologies, African governments have adopted strategies to mobilise resources to digitalise information systems in health structures. Projects to implement digital health information systems are thus a hot topic. Although more or less functional in most developing countries, HIS are currently described as poorly performing for several reasons, including the fact that they use anarchic systems where much data is recorded on paper charts, files and registers [6][7][8].

According to a survey carried out in 15 African French-speaking countries, HIS digital remains the least developed component. In most of these countries, the modernisation of the health system does not sufficiently integrate the HIS computerisation. The few initiatives observed are more focused on hospital accounting management than on the management of medical information [9].

In Burundi, many actions have been carried out to strengthen the health information system, including the implementation of DHIS 2 (District Health Information Software version 2), which is a software package for managing routine data collected in health facilities. This software, which is only limited to the management of aggregated data, should be completed by other software that allows for the management of computerised patient records, resource management and networking within a hospital. It is in this context that an HIS project, using OpenClinic GA software, was initiated in Burundi hospitals [10].

Before the computerization project, the tools used to manage the HIS were largely paper-based (patient medical records, follow-up sheets, registers, monthly activity reports, etc.), with the direct consequence that the quality of the data produced was poor. An evaluation of the national health information system using the Health Metrology Network (HMN) tool in 2008 showed that health data sources based on registration and notification of diseases within health facilities had a low score of $43 \%$ [11]. Two other routine health information system assessments, carried out in 2013 and 2016 with the Performance of Routine Information Systems (PRISM) tool, showed that the overall performance at the level of health facilities (without distinguishing between hospitals and health centres) improved significantly from 59\% to $63 \%$ in 2016. Nevertheless, it was noted that the overall quality of data decreased from the national level to the health facilities, which partly reflected the level of complexity and workload of providers at the health facility level [12][13].

In response to the challenges identified by the various evaluations, the OpenClinic GA software was implemented. This hospital computerization project was financed by ENABEL in its component which aimed to support the Ministry of Public Health and the Fight against AIDS in the modernization of the hospital information system. The approach of the computerisation project was to start with a pilot phase in 4 hospitals representative of the 3 levels of the health pyramid: the district, regional and national levels. In the logic of gradually extending the computerisation of hospitals in 2017, seven hospitals were equipped and started using the OpenClinic GA software. The chosen software has been developed by a Belgian company MXS SA since 2006 and can be redistributed or modified, commercial versions are also available. The main modules of the software concern the patient's administrative file, the patient's financial file, the patient's medical file, the health insurance, the cash registers, the pharmacy (including stock), the laboratory, radiology, health statistics in the form of periodic reports, human resources, the clinical thesaurus with validated coding aid for ICD-10, SNOMED and multimedia supports (images, video, audio) [14].

Since the implementation of the computerization project, no evaluation has been made. In order to explore the state of the digital hospital information system, it was important to evaluate the six components (resources, indicators, sources, management, information products and data dissemination and use) of the HIS according Health Metrology Network method [15], to compare the performance of the HIS according to the reference levels of the study hospitals and the period of introduction of the electronic medical record.

\section{Materials and Methods}

\subsection{Type and Period of Study}

This was a descriptive cross-sectional study using the assessment tools, carried out in October 2019 in 11 hospitals equipped with an HIS based on OpenClinic GA software.

\subsection{Target Population}

Eleven hospitals equipped with an HIS based on OpenClinic GA software and belonging to the 3 levels of the health pyramid in Burundi, were included in the study, 4 of which were computerized in 2015 and 7 of which were computerized in 2017. Four of them belonged to the 3rd referral level (district), two to the 2nd referral level (region) and 5 to the 1st referral level (national). They were all beneficiaries of a computerization project financed by ENABEL.

In each hospital, 3 key informants chosen for their areas 
of expertise in relation to the six components of the system were interviewed. They were the director in charge of care, the head of the nursing service and the person in charge of the health information system.

\subsection{Data Collection Tools}

The HIS assessment was carried out using the RMS tool [15] based on a semi-quantitative method, which is useful for identifying weaknesses and strengths in the following six components: resources, indicators, data sources, data management, information product and information dissemination and use. The RMS tool, usually intended for the assessment of national information systems, has been adapted for use in the hospital context. The national adaptation and validation of the RMS tool was carried out by a team of national HIS experts. Thus, out of 197 questions or items in the basic WHO model, 116 items were retained in the adapted model (Table 1).

Table 1. Distribution of items from the full WHO Health Metrics Network (HMN) model and the model adapted for Burundi Hospital Information System

\begin{tabular}{ccc}
\hline \multirow{2}{*}{ Components } & \multicolumn{2}{c}{ Number of questions (items) } \\
\cline { 2 - 3 } & HMN tool & Adapted HMN tool \\
\hline Resources & 25 & 18 \\
\hline Indicators & 5 & 4 \\
\hline Sources of data & 83 & 56 \\
\hline Data management & 5 & 5 \\
\hline Information products & 69 & 24 \\
\hline Dissemination and use & 10 & 9 \\
\hline Total & $\mathbf{1 9 7}$ & $\mathbf{1 1 6}$ \\
\hline
\end{tabular}

For each item, the assessment was based on scores ranging from zero to three $(0=$ not at all satisfactory; $1=$ exists but not satisfactory; $2=$ satisfactory and $3=$ very satisfactory). The average score for each component was related to the maximum score of three. The calculated scores were thus converted into quartiles: $0-25 \%=\mathrm{Q} 1$ not at all satisfactory, $26-50 \%=\mathrm{Q} 2$ present but not satisfactory, $51-75 \%=\mathrm{Q} 3$ satisfactory and $76-100 \%=\mathrm{Q} 4$ very satisfactory.

In each hospital, 3 key informants chosen for their areas of expertise in relation to the six components of the system were interviewed. The answer selected was the result of a consensus. In the event of a discrepancy between the 3 key informants, all the answers were used to calculate an average score.

\subsection{Data Collection Tools}

Data entry, processing and analysis were carried out using the Excel tool transposed into the format of the RMS tool. Scores and quartiles were calculated for each of the 11 study hospitals, for each component, before calculating them by hospital group according to the level of membership in the health pyramid and according to the date of implementation of the digital HIS. The comparison of average scores between hospital groups was made using the Wilcoxon-Mann-Whitney test.

The first comparison variable was the year of start of use of the electronic patient record with two modalities namely (i) computerized hospitals in 2015 and (ii) computerized hospitals in 2017. The second comparison variable was the level of referral of study hospitals with three modalities, namely (i) 1st referral hospitals (district); (ii) 2nd referral hospitals (regional) and (iii) 3rd referral hospitals (national).

\subsection{Study Limit}

The Health Metric Network's tool [15] uses a normative evaluation approach and does not explore the causes of the inadequacies observed. The results of this evaluation should be supplemented by another study that could describe the factors explaining the deficiencies observed in the six components of the HIS.

\subsection{Ethical Considerations}

The research protocol has been submitted to the National Health Research Ethics Committee for approval. In addition, the authorisation to collect the data in the selected hospitals was given by the Ministry of Public Health and the Fight against AIDS. The directors of the hospitals concerned by this survey have given their agreement. For the providers who were identified to be interviewed, free and informed consent was given, and the interviews were carried out respecting anonymity and confidentiality. For the patient files that were used, the identity of the patients (surname and first name, address, telephone number, etc.) was not recorded on the data collection tools.

\section{Results}

The presentation sequence of results starts with the evaluation results of the Hospital Information System as a whole and ends with the evaluation results of the six components of the Health Metrology Network: resources, indicators, data sources, data management, information production and information dissemination and use.

\subsection{Overall Result for the Hospital Information System}

For all the hospitals benefiting from digital HIS, the average calculated from the scores obtained by each of the six components reached a satisfactory level of $56 \%$.

Generally speaking, five of the six components of the information system were satisfactory, with a score of between $51 \%$ and $67 \%$, falling in the third quartile (Q3): resources $(61 \%$; Q3), indicators $(60 \%$; Q3), data management $(51 \%$; Q3), information production $(55 \%$; 
Q3), and dissemination and use of information (67\%; Q3)). One component had a score in the 2nd quartile (Q2), the data source component with the lowest score of $41 \%$ (figure 1).

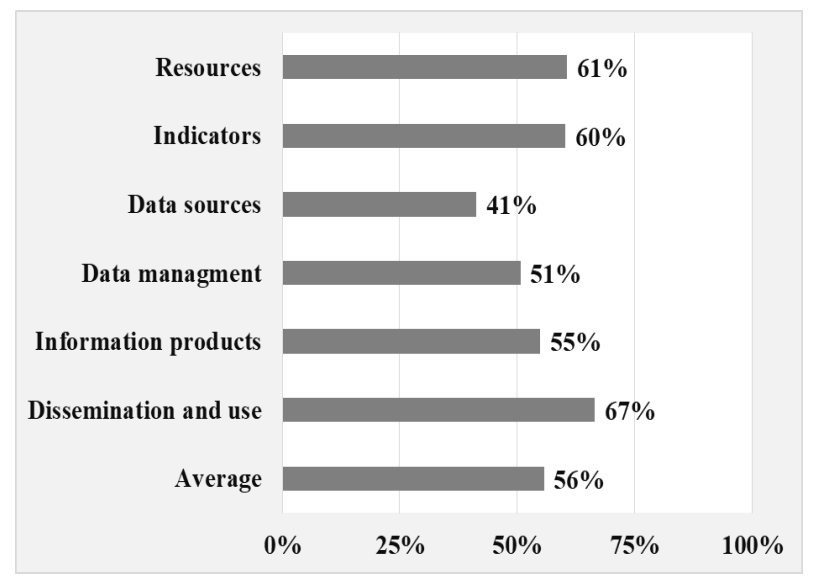

Figure 1. Comparison of HIS performance scores by component of HIS in Burundi, $2019(\mathrm{n}=11)$

\subsection{Detailed Results for Each of the Six Components of the HIS}

The "resources" component of the HIS scored 61\% (Q3). More specifically, the availability of HIS equipment had a score of $84 \%$, coordination, planning and policies $50 \%(\mathrm{Q} 3)$ and the human resources and financing HIS sub-component did not exceed a score of $48 \%(\mathrm{Q} 2)$.
For the "indicators" component, the selection, definition, production and dissemination of core indicators in accordance with national guidelines achieved a satisfactory score of $60 \%(\mathrm{Q} 3)$.

The score achieved by the "data sources" component was $41 \%$, included in the second quartile Q2. In detailing the score achieved by the sub-components, the recording of disease data and resource data had achieved a similar score of $68 \%(\mathrm{Q} 3)$. The scores obtained by the other sub-components were $46 \%$ (Q2) for the health services data recording and management system; 16\% (Q1) for population surveys and $8 \%(\mathrm{Q} 1)$ for the availability and use of census data by the hospital.Q1. The civil status sub-component did not assess (table 2).

The "data management" component scored 51\% (Q3) in the third quartile $(\mathrm{Q} 3)$.

The "information production" component measures the hospital's capacity to produce morbidity and mortality indicators on the one hand and health system indicators on the other had achieved a score of 55\% (Q3). The score obtained by the sub-component production of morbidity and mortality indicators was $49 \%$ (Q2), while the production of health system indicators scored $61 \%(\mathrm{Q} 3)$.

The "dissemination and use of information" component scored $67 \%(\mathrm{Q} 3)$. At the level of its sub-components the use of information for planning and priority setting achieved a score of $75 \%(\mathrm{Q} 3)$; the use of information for resource allocation achieved a score of $67 \%(\mathrm{Q} 3)$ and the analysis of information $60 \%(\mathrm{Q} 3)$.

Table 2. Item's scores (\%) of "data sources" component of the HIS in 2019

\begin{tabular}{|c|c|c|c|c|c|}
\hline Sources of data & Contents & Capacities practices & Dissemination & Integration use & Average \\
\hline Census & 9 & 10 & 0 & 6 & 8 \\
\hline Population surveys & 16 & 26 & 9 & 5 & 16 \\
\hline Monitoring system & 65 & 73 & 25 & 80 & 68 \\
\hline Health services & 55 & 36 & 69 & 43 & 46 \\
\hline Resources & 84 & 64 & 49 & 50 & 68 \\
\hline Average & & & & 41 \\
\hline
\end{tabular}

Legend :

not at all satisfactory

$0-25 \%(\mathrm{Q} 1)$ exists but not satisfactory

$26-50 \%(\mathrm{Q} 2)$ satisfactory

51-75\% (Q3) very satisfactory

$>=76(\mathrm{Q} 4)$ 


\subsection{Comparison of HIS Performance between Hospitals}

According to the period when EMR was first used, the seven (7) hospitals computerised in 2017 had an overall score of $57 \%(\mathrm{Q} 3)$ compared to $53 \%(\mathrm{Q} 3)$ of the four (4) computerised in 2015. For the resources, indicators, information products and information dissemination components, a significant gap between the two groups of hospitals was identified in favour of computerized hospital in 2017. The scores for all components fall between the 2 nd and 3 rd quartile regardless of the period when the EMR is first used (figure 2).

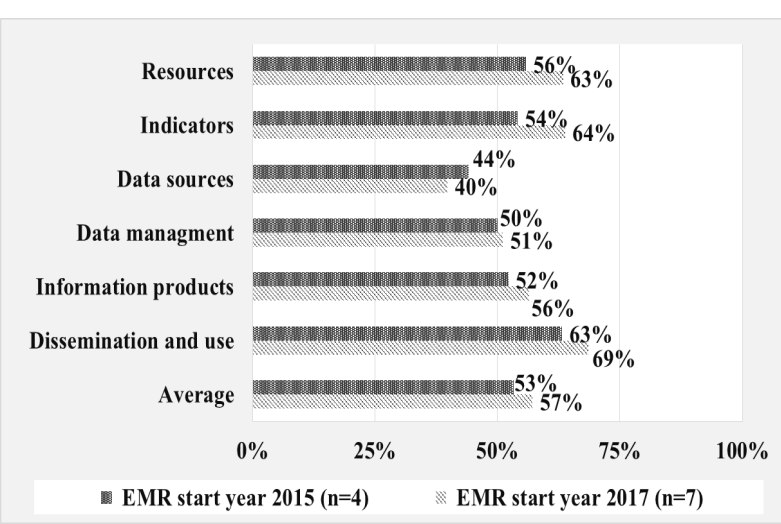

Figure 2. Comparison of HIS performance scores according to the year of start using EMR, 2019

Depending on the level of referral of the study hospitals, the analysis also covered five (5) hospitals with a 1st referral (district); two (2) hospitals with a 2nd referral (regional) and four (4) hospitals with a 3rd referral (national). The district hospitals had an overall score of $61 \%$ (Q3), followed by national hospitals with a score of $52 \%(\mathrm{Q} 3)$. The group of regional hospitals had a lowest score of $50 \%$. The district hospitals significantly outperformed the others for all three components: indicators $(68 \%, \mathrm{Q} 3)$, information production $(70 \%, \mathrm{Q} 3)$ and dissemination and use of information $(72 \%, \mathrm{Q} 3)$ (figure 3).

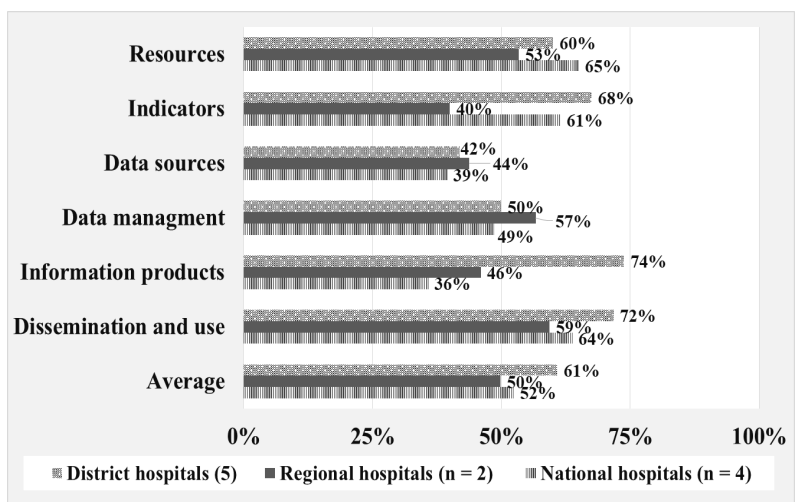

Figure 3. Comparison of HIS performance scores according to the reference level of study hospitals, 2019

Comparison of average HIS performance scores between groups of hospitals using the Wilcoxon-Mann-Whitney test showed that there was no statistically significant difference between the group of hospitals that introduced the use of EMR in 2015 and those that introduced EMR in 2017 on the one hand and district hospitals compared to regional and national hospitals on the other hand (table 3).

\section{Discussion}

The HIS evaluation of the eleven hospitals carried out using RMS adapted tools made it possible to measure the HIS performance in the context of the use of EMR and to identify the main weak points that would require concrete actions to achieve the expected effects of the project to computerise the patient file.

\subsection{Evaluation of the Six Components}

The average score for all eleven hospitals and all six components was $56 \%$ and was at the lower limit of the third quartile (Q3). The data source component had the lowest score of $41 \%$ falling in the 2nd quartile (Q2), while the dissemination and use component had the highest score of $67 \%$ (Q3) compared to the other components.

Table 3. Comparison of performance scores by study hospital characteristics

\begin{tabular}{|c|c|c|c|c|c|c|}
\hline \multirow{2}{*}{$\begin{array}{l}\text { Components } \\
\text { of the HIS }\end{array}$} & \multicolumn{2}{|c|}{ Year start of use EPR } & \multirow{2}{*}{ p-value } & \multicolumn{2}{|c|}{ Reference level } & \multirow{2}{*}{ p-value } \\
\hline & $2015(n=4)$ & $2017(n=7)$ & & District $(n=5)$ & National /Regional $(n=6)$ & \\
\hline Resources & $56 \%$ & $63 \%$ & 0,5708 & $60 \%$ & $57 \%$ & 0,8551 \\
\hline Indicators & $54 \%$ & $64 \%$ & 0,7042 & $68 \%$ & $54 \%$ & 0,5209 \\
\hline Sources of data & $44 \%$ & $40 \%$ & 0,7055 & $42 \%$ & $43 \%$ & 0,8551 \\
\hline Data management & $50 \%$ & $51 \%$ & 0,9999 & $50 \%$ & $53 \%$ & 0,9999 \\
\hline Information products & $52 \%$ & $56 \%$ & 0,9999 & $74 \%$ & $60 \%$ & 0,1003 \\
\hline Dissemination and use & $63 \%$ & $69 \%$ & 0,7055 & $72 \%$ & $66 \%$ & 0,4652 \\
\hline Average & $53 \%$ & $57 \%$ & 0,7055 & $60 \%$ & $55 \%$ & 0,1441 \\
\hline
\end{tabular}


Due to the low score recorded for the data source component, specifically for the sub-component on the availability and use of census data by the hospital, it was noted that the calculation of indicators requiring population projections is not a common practice in the hospital (see the information products component, the section on the calculation of morbidity and mortality indicators with a score of $49 \%$ ). In addition, the indicators whose numerator and denominator are all produced by the hospital are regularly calculated (see the information products component, section on the calculation of health system indicators with a score of $61 \%$ ). Another study had noted the same problem of the availability and use of target populations by hospitals [16]. The current context pointing to the persistence of strong territorial and social inequalities in health should lead hospitals to take into account more strongly the characteristics of the populations in the health territories they serve. This information, which also serves as denominators for the calculation of indicators, is produced within the framework of censuses.

The sub-component of conducting hospital client surveys was judged unsatisfactory with a score of $16 \%$. Irregularity in the conduct of the studies was observed and the capacity of the hospital staff to conduct the studies was judged insufficient. Although many other studies or surveys can be carried out among patients in the hospital, particularly in the context of using an electronic medical record where data is available [17][18][19], one of the types of survey recommended in hospitals and made mandatory in some countries is the client satisfaction survey. The low score found for the sub-component conducting hospital client surveys is only a true picture of what is happening in most hospitals in developing countries where research that leads mainly to action is not a priority [20][21][22].

The sub-component "health services data recording and management system" scored $46 \%$. The inadequacies observed stem from the interoperability problems that persist between the software for managing the patient record (OpenClinic GA) and the software for managing aggregate data (DHIS 2). The problem of interoperability of the applications used in healthcare structures is a major issue because the software used in the EMR implementation process is developed without prior analysis of the system already in place, using informatics heterogeneous tools, methods and procedures [23]. It was also noted that the aspects explored that had an impact on this sub-component were related to the low competence of the staff in charge of data management in the hospital's departments and the lack of a training plan aimed at in-service capacity building and support for the higher level. This situation related to the lack of human resource skills to manage data was found in other evaluations conducted in Burkina Faso and Côte d'Ivoire [24][25].

For the data management component which had a score at the lower limit of Q3 (51\%), low scores were noted for the sub-components related to database management and metadata use. Indeed, the database management problem stems from the fact that the software used to manage the medical record is found only at the hospital level, other levels of the health system that should accompany and work closely with the hospital have no access to this software and there is no policy defining the role of the actors at the other levels. Health Information System standards recommend that the software implemented in health care structures should be found at other levels of the health system with a utility to which various actors have access according to their respective roles, in strict compliance with the principles of ethics and confidentiality of patient data [15][26].

Concerning the use of metadata, the ideal situation would be to find in the hospital a metadata dictionary or a document of standards and procedures for the management of the Health Information System that provides the definition of variables, the methods of data collection and calculation of indicators, the periodicity, the analysis techniques to be used and the possible systematic errors to be avoided. This evaluation showed that no hospital had this dictionary. The same consistent pattern of absence from the documents of the standards for managing the Health Information System in health care facilities was found elsewhere [15][21][24].

\subsection{Comparison of HIS Performance between Hospitals}

The overall HIS performance score was in favour of computerised hospitals in 2017. The experience of the hospitals in the pilot phase (computerised in 2015) has undoubtedly contributed to improvements in the process of extending the implementation of the software.

The comparison of HIS performance between the three types of hospitals according to their reference level showed that the district hospitals had the highest score compared to the others. This could be explained by the fact that the district level in general, and the district hospital in particular, is the most supported in terms of technical and financial support from partners, health programmes and projects [27].

Using the non-parametric Wilcoxon-Mann-Whitney test adapted to compare the distribution of small subjects, the average scores of the hospital groups were compared. There were no statistically significant differences observed according to the year of introduction of the EMR and the reference level of the study hospitals. These results do not allow to conclude on the existence of an association between hospital characteristics (number of years of EMR use, reference level,...) and HIS performance. Furthermore, it would be relevant to carry out in-depth research on a sufficient number of hospitals to confirm or disprove this hypothesis. 


\subsection{Recommendation for Future Research}

In order to explore the factors that explain the inadequacies observed in the six components of the HIS, the results of this evaluation should be supplemented by another study based on a qualitative and quantitative approach among users, managers at different levels of the health system and technical and financial partners.

In this sense, an evaluation of the implementation, the implementation of the computerisation project and the adherence, perceptions and satisfaction of the providers is underway, the results will allow to complete the information produced in this article.

\section{Conclusions}

The Hospital Information System (HIS) evaluation based on the RMS tools made it possible to assess its performance at the level of the eleven (11) hospitals. This evaluation showed that five components (resources, indicators, data management, information production and dissemination and use) were satisfactory with a score ranging from $51 \%$ to $67 \%$. The data source component had the lowest score of $41 \%$.

The HIS performance improvement, more particularly the effectiveness and management of the computerised medical record, is determined by all the resources and actions taken during the implementation process of the computerisation project, the level of collaboration between the actors at different levels of the health system, the support measures aimed at a good integration of the new system, the policies and strategies in force in terms of HIS and the leadership of the decision-makers [28].

\section{Acknowledgements}

To the General Directorate of the National Institute of Public Health (NIPH) in Bujumbura, to the managers of the NIPH Project funded by European Union for technical and financial support, and to the data managers and computer specialists of the Ministry of Public Health and the Fight against AIDS for data collection.

\section{REFERENCES}

[1] S. Chougrani, S. Ouhadj, and F. Agag, "Evaluation of the Oran uniersity hospital information system," Sante Publique (Paris)., vol. 25, no. 5, pp. 617-626, 2013.

[2] C. AbouZahr and T. Boerma, "Health information systems: the foundations of public health," Bull. World Health Organ., vol. 83, no. 8, pp. 578-583, 2005.

[3] A. A. Esfahani et al., "An evaluation model for the implementation of hospital information system in public hospitals using multi-criteria-decision-making (MCDM) approaches," Int. J. Eng. Technol., vol. 7, no. 1, pp. 1-18, 2018.

[4] C. S. Kruse, C. Kristof, B. Jones, E. Mitchell, and A. Martinez, "Barriers to Electronic Health Record Adoption: a Systematic Literature Review," J. Med. Syst., vol. 40, no. 12, 2016.

[5] D. Dupont, A. Beresniak, D. Kalra, P. Coorevits, and G. De Moor, "Intérêt des dossiers de santé électroniques hospitaliers pour la recherche clinique," Médecine/Sciences, vol. 34, no. 11, pp. 972-977, 2018.

[6] Y. G. Ahanhanzo, J. Saizonou, A. Wodon, B. Dujardin, M. Wilmet-Dramaix, and M. Makoutodé, "Health workers involvement for data quality improvement in Benin," Santé Publique (Paris)., vol. 27, no. 2, pp. 241-248, 2015.

[7] M. Cucciniello, I. Lapsley, G. Nasi, and C. Pagliari, "Understanding key factors affecting electronic medical record implementation: A sociotechnical approach," $B M C$ Health Serv. Res., vol. 15, no. 1, 2015.

[8] K. E. M. Msiska, A. Kumitawa, and B. Kumwenda, "Factors affecting the utilisation of electronic medical records system in Malawian central hospitals," Malawi Med. J., vol. 29, no. 3, 2017.

[9] C. O. Bagayoko, H. Müller, and A. Geissbuhler, "Assessment of Internet-based tele-medicine in Africa (the RAFT project)," Comput. Med. Imaging Graph., vol. 30, no. 6-7, pp. 407-416, 2006.

[10] République du Burundi. Ministère de la Santé Publique et de la lutte contre le Sida, "Plan National de Développement de l'Informatique de la Santé ( PNDIS ),” 2015.

[11] République du Burundi. Ministère de la Santé Publique et de la lutte contre le Sida, "Rapport d'évaluation du Système National d'Information Sanitaire du Burundi," 2008.

[12] République du Burundi. Ministère de la Santé Publique et de Lutte contre le Sida, "Rapport d'évaluation du systeme d' information sanitaire de routine par l' approche et les outils PRISM,” 2015.

[13] République du Burundi. Ministère de la Santé Publique et de la lutte contre le Sida, "Rapport d'Evaluation des Performances du Système National d'Information Sanitaire de Routine au Burundi," 2016.

[14]F. Verbeke, OpenClinic v3.50.11 Manuel utilisateur. 2010.

[15] World Health Organization-WHO, Framework and standards for country health information systems. Health Metrics Network. 2008.

[16] A. Delas, L'hôpital public, un nouvel acteur territorial entre aménagement sanitaire et rivalités stratégiques, vol. 143, no. 4, pp 89-119, 2011.

[17] H. Lefebvre, O. Roy, S. Sahtali, and M. Rothan-Tondeur, "Liens entre recherche et pratique: échange des savoirs, utilisation des données probantes et renouvellement des pratiques," Rech. Soins Infirm., vol. № 114, no. 3, pp. 31-45, 2013.

[18]É. Péchillon, "Open access to health data : Can the law guarantee all risks of any excesses in the use of the information?," Inf. Psychiatr., vol. 91, no. 8, pp. 645-649, 
2015.

[19] P. Campanella et al., "The impact of electronic health records on healthcare quality: A systematic review and meta-analysis," in European Journal of Public Health, 2016, vol. 26 , no. 1 .

[20] L. Gerbaud, S. Antoniotti, P. Auquier, P. Yvonne, and S. F. S. P. S. Publique, "The increase use of hospitals discharge questionnaires, a study involing 7 hospital centres," Santé Publique (Paris)., vol. 14, no. 1, pp. 21-30, 2002.

[21] I. E. Obi, A. C. Ndu, K. A. Agu, B. I. Omotowo, C. C. Agunwa, and A. C. Idoko, "Patient satisfaction with services at a tertiary hospital in south-east Nigeria," Malawi Med. J., vol. 30, no. 4, pp. 270-275, 2018.

[22] L. Ndi, "Perceptions and Therapeutic Challenges in the Management of Chronic STIs in Africa: Comparing HIV and Hepatitis B in the University Teaching Hospital Yaounde, Cameroon," Univers. J. Public Heal., vol. 7, no. 1, pp. 1-8, 2019. DOI: 10.13189/ujph.2019.070101.

[23] O. Iroju, A. Soriyan, I. Gambo, and J. Olaleke, "Interoperability in Healthcare: Benefits, Challenges and Resolutions," Int. J. Innov. Appl. Stud., vol. 3, no. 1, pp. 262-
$270,2013$.

[24] S. T. Sory, A. Baguiya, A. Coulibaly, S. Kouanda, and B. Sondo, "Evaluation de la performance du système d'information hospitalier du Centre Hospitalier Universitaire Yalgado Ouédraogo de Ouagadougou, Burkina Faso," Sci. Tech. Sci. la santé, vol. 40, no. 1, pp. 135-150, 2017.

[25] République de Côte d'Ivoire. Ministère de la Santé et de l'Hygiène Publique, "Evaluation du Système d'Information de gestion de Côte d'Ivoire.," 2004.

[26] World Health Organization-WHO, Legal frameworks for eHealth: based on the results of the 2and Global e-Health survey, Global Obs., vol. 5. Geneva, 2012.

[27]F. J. T., K. O., and B. O. I., "Spatial Analysis of Distribution Patterns of Healthcare Facilities in Osun State, Nigeria," Univers. J. Public Heal., vol. 5, no. 7, pp. 331-344, 2017. DOI: 10.13189/ujph.2017.050701.

[28] M. Béjean, F. Kletz, and J.-C. Moisdon, "Création de valeur organisationnelle et technologies de 1 ' information à 1 ' hôpital: le cas du dossier patient informatisé Mots-clés," Gest. Manag. public, vol. 6, no. 4, pp. 9-24, 2018. 